\title{
High-order conservative Crank-Nicolson scheme for regularized long wave equation
}

Kelong Zheng ${ }^{1}$ and Jinsong $\mathrm{Hu}^{2^{*}}$

"Correspondence: hujs7758@163.com

${ }^{2}$ School of Mathematics and Computer Engineering, Xihua University, Chengdu, Sichuan 610039, P.R. China

Full list of author information is available at the end of the article

\begin{abstract}
Numerical solution for the regularized long wave equation is studied by a new conservative Crank-Nicolson finite difference scheme. By the Richardson extrapolation technique, the scheme has the accuracy of $O\left(\tau^{2}+h^{4}\right)$ without refined mesh. Conservations of discrete mass and discrete energy are discussed, and existence of the numerical solution is proved by the Browder fixed point theorem. Convergence, unconditional stability as well as uniqueness of the solution are also derived using energy method. Numerical examples are carried out to verify the correction of the theory analysis.
\end{abstract}

MSC: $65 \mathrm{M} 06 ; 65 \mathrm{~N} 30$

Keywords: RLW equation; conservative difference scheme; Richardson extrapolation; stability; convergence

\section{Introduction}

Consider the initial boundary value problem for the regularized long wave (RLW) equation

$$
u_{t}+u_{x}+u u_{x}-u_{x x t}=0, \quad x \in\left(x_{L}, x_{R}\right), t \in(0, T]
$$

with an initial condition

$$
u(x, 0)=u_{0}(x), \quad x \in\left[x_{L}, x_{R}\right]
$$

and a boundary condition

$$
u\left(x_{L}, t\right)=u\left(x_{R}, t\right)=0, \quad t \in[0, T],
$$

where $u_{0}(x)$ is a given known function. The RLW equation is originally introduced as an alternative to the Korteweg-de Vries (KdV) equation to describe the behavior of the undular bore by Peregrine [1] and plays a very important role in physics media, since it describes phenomena with weak nonlinearity and dispersion waves, including nonlinear transverse waves in shallow water, ion-acoustic and magneto hydrodynamic waves in plasma and phonon packets in nonlinear crystals. When it is used to model waves generated in a shallow water channel, the variables are normalized in the following way: distance $x$ and water elevation $u$ are scaled to the water depth $h$, and time $t$ is scaled to $\sqrt{\frac{h}{g}}$, where $g$ is the ac-

(92013 Zheng and Hu; licensee Springer. This is an Open Access article distributed under the terms of the Creative Commons Attribution License (http://creativecommons.org/licenses/by/2.0), which permits unrestricted use, distribution, and reproduction in any medium, provided the original work is properly cited. 
celeration due to gravity. The physical boundary requires

$$
u \rightarrow 0, \quad \text { as }|x| \rightarrow \infty
$$

So, if $-x_{L} \gg 0$ and $x_{R} \gg 0$, problems (1.1)-(1.3) is in accordance with the Cauchy problem of equation (1.1). The RLW equation has the following conserved laws,

$$
Q(t)=\int_{x_{L}}^{x_{R}} u(x, t) d x=\int_{x_{L}}^{x_{R}} u_{0}(x) d x=Q(0)
$$

and

$$
E(t)=\|u\|_{L_{2}}^{2}+\left\|u_{x}\right\|_{L_{2}}^{2}=\left\|u_{0}\right\|_{L_{2}}^{2}+\left\|\left(u_{0}\right)_{x}\right\|_{L_{2}}^{2}=E(0),
$$

where $Q(0)$ and $E(0)$ are two positive constants which relate to the initial condition.

Existence and uniqueness of the solution of the RLW equation are given in [2]. Its analytical solution was found [3] under restricted initial and boundary conditions, and, therefore, it became interesting from a numerical point of view. Some numerical methods for the solution of the RLW equation such as variational iteration method $[4,5]$, finite-difference method [6-8], Fourier pseudospectral method [9], finite element method [10-13], collocation method [14] and adomian decomposition method [15] have been introduced in many works. In [16], $\mathrm{Li}$ and $\mathrm{Vu}$-Quoc pointed out that 'in some areas, the ability to preserve some invariant properties of the original differential equation is a criterion to judge the success of a numerical simulation.' Meanwhile, Zhang et al. [17] thought that the conservative difference schemes perform better than the nonconservative ones, and the non-conservative difference schemes may easily show nonlinear 'blow-up. Hence, constructing a conservative difference scheme for the numerical solution of the nonlinear partial differential equation is quite significant. In this paper, coupled with the Richardson extrapolation, a two-level nonlinear Crank-Nicolson finite difference scheme for problems (1.1)-(1.3), which has the accuracy of $O\left(\tau^{2}+h^{4}\right)$ without refined mesh is proposed. The scheme simulates two conserved quantities (1.5) and (1.6) well, respectively. Moreover, priori estimate, existence and uniqueness of the numerical solutions are discussed. Convergence and unconditional stability of the scheme are also proved.

The outline of the paper is as follows. In Section 2, a nonlinear conservative difference scheme is proposed. In Section 3, we prove the existence of the difference solution by the Browder fixed point theorem. Priori estimate, convergence and stability are proved in Section 4, and numerical experiments to verify the theoretical analysis are reported in Section 5.

\section{Nonlinear finite difference scheme}

As usual, let $h=\frac{x_{R}-x_{L}}{J}$ be the step size for the spatial grid such that $x_{j}=x_{L}+j h$ $(j=-1,0,1, \ldots, J, J+1)$. Let $\tau$ be the step size for the temporal direction $t_{n}=n \tau(n=$ $0,1,2, \ldots, N), N=\left[\frac{T}{\tau}\right]$. Denote $u_{j}^{n} \approx u\left(x_{j}, t_{n}\right)$ and

$$
Z_{h}^{0}=\left\{u=\left(u_{j}\right) \mid u_{-1}=u_{0}=u_{J}=u_{J+1}=0, j=0,1,2, \ldots, J\right\} .
$$


Define

$$
\begin{array}{ll}
\left(u_{j}^{n}\right)_{x}=\frac{u_{j+1}^{n}-u_{j}^{n}}{h}, & \left(u_{j}^{n}\right)_{\bar{x}}=\frac{u_{j}^{n}-u_{j-1}^{n}}{h}, \quad\left(u_{j}^{n}\right)_{\hat{x}}=\frac{u_{j+1}^{n}-u_{j-1}^{n}}{2 h}, \\
\left(u_{j}^{n}\right)_{\ddot{x}}=\frac{u_{j+2}^{n}-u_{j-2}^{n}}{4 h}, & \left(u_{j}^{n}\right)_{t}=\frac{u_{j}^{n+1}-u_{j}^{n}}{\tau},
\end{array}
$$

In the paper, $C$ denotes a general positive constant which may have different values in different occurrences.

Lemma 2.1 For a mesh function $u \in Z_{h}^{0}$, we have

$$
\left\|u_{\ddot{x}}\right\|^{2} \leq\left\|u_{\hat{x}}\right\|^{2} \leq\left\|u_{x}\right\|^{2} .
$$

Proof Obviously,

$$
\begin{aligned}
& \left(u_{j}\right)_{\ddot{x}}=\frac{u_{j+2}-u_{j-2}}{4 h}=\frac{1}{2}\left(\frac{u_{j+2}-u_{j}}{2 h}+\frac{u_{j}-u_{j-2}}{2 h}\right)=\frac{1}{2}\left(\left(u_{j+1}\right)_{\hat{x}}+\left(u_{j-1}\right)_{\hat{x}}\right), \\
& \left(u_{j}\right)_{\hat{x}}=\frac{u_{j+1}-u_{j-1}}{2 h}=\frac{1}{2}\left(\frac{u_{j+1}-u_{j}}{h}+\frac{u_{j}-u_{j-1}}{h}\right)=\frac{1}{2}\left(\left(u_{j}\right)_{x}+\left(u_{j}\right)_{\bar{x}}\right) .
\end{aligned}
$$

Since $\forall u \in Z_{h}^{0}$, we have $\left\|u_{x}\right\|^{2}=\left\|u_{\bar{x}}\right\|^{2}$. By Cauchy-Schwarz inequality, we get

$$
\begin{aligned}
& \left\|u_{\ddot{x}}\right\|^{2}=\frac{1}{4} h \sum_{j=0}^{J}\left(\left(u_{j+1}\right)_{\hat{x}}+\left(u_{j-1}\right)_{\hat{x}}\right) \cdot\left(\left(u_{j+1}\right)_{\hat{x}}+\left(u_{j-1}\right)_{\hat{x}}\right) \leq\left\|u_{\hat{x}}\right\|^{2}, \\
& \left\|u_{\hat{x}}\right\|^{2}=\frac{1}{4} h \sum_{j=0}^{J}\left(\left(u_{j}\right)_{x}+\left(u_{j}\right)_{\bar{x}}\right) \cdot\left(\left(u_{j}\right)_{x}+\left(u_{j}\right)_{\bar{x}}\right) \leq\left\|u_{x}\right\|^{2} .
\end{aligned}
$$

The following Crank-Nicolson conservative difference scheme for problems (1.1)-(1.3) is considered,

$$
\begin{aligned}
& \left(u_{j}^{n}\right)_{t}-\frac{4}{3}\left(u_{j}^{n}\right)_{x \bar{x} t}+\frac{1}{3}\left(u_{j}^{n}\right)_{\hat{x} \hat{x} t}+\frac{4}{3}\left(u_{j}^{n+\frac{1}{2}}\right)_{\hat{x}}-\frac{1}{3}\left(u_{j}^{n+\frac{1}{2}}\right)_{\ddot{x}} \\
& \quad+\frac{4}{9}\left\{\left(u_{j}^{n+\frac{1}{2}}\right)\left(u_{j}^{n+\frac{1}{2}}\right)_{\hat{x}}+\left[\left(u_{j}^{n+\frac{1}{2}}\right)^{2}\right]_{\hat{x}}\right\} \\
& \quad-\frac{1}{9}\left\{u_{j}^{n+\frac{1}{2}}\left(u_{j}^{n+\frac{1}{2}}\right)_{\ddot{x}}+\left[\left(u_{j}^{n+\frac{1}{2}}\right)^{2}\right]_{\ddot{x}}\right\}=0, \quad j=1,2, \ldots, J-1 ; n=1,2, \ldots, N-1, \\
& u_{j}^{0}=u_{0}\left(x_{j}\right), \quad 0 \leq j \leq J, \\
& u^{n} \in Z_{h}^{0}, \quad n=0,1,2, \ldots, N .
\end{aligned}
$$

From boundary condition (1.3), and physical boundary (1.4), discrete boundary condition (2.3) is reasonable. Based on scheme (2.1)-(2.3), the discrete versions of (1.5) and (1.6) are obtained as follows. 
Theorem 2.1 Scheme (2.1)-(2.3) admits the following invariants, i.e.,

$$
\begin{aligned}
& Q^{n}=h \sum_{j=0}^{J} u_{j}^{n}=Q^{n-1}=\cdots=Q^{0}, \\
& E^{n}=\left\|u^{n}\right\|^{2}+\frac{4}{3}\left\|u_{x}^{n}\right\|^{2}-\frac{1}{3}\left\|u_{\hat{x}}^{n}\right\|^{2}=E^{n-1}=\cdots=E^{0}
\end{aligned}
$$

for $n=1,2, \ldots, N$.

Proof Multiplying (2.1) with $h$, then summing up for $j$ from 1 to $J-1$, by boundary condition (2.3) and formula of summation by parts [18], we have

$$
h \sum_{j=0}^{J}\left(u_{j}^{n}\right)_{t}=0 .
$$

By the definition of $Q^{n},(2.4)$ is obtained from (2.6).

Taking the inner product of (2.1) with $2 u^{n+\frac{1}{2}}$, according to boundary condition (2.3), we get

$$
\begin{aligned}
& \left\|u^{n}\right\|_{t}^{2}+\frac{4}{3}\left\|u_{x}^{n}\right\|_{t}^{2}-\frac{1}{3}\left\|u_{\hat{x}}^{n}\right\|_{t}^{2}+\frac{8}{3}\left\langle u_{\hat{x}}^{n+\frac{1}{2}}, u^{n+\frac{1}{2}}\right\rangle-\frac{2}{3}\left\langle u_{\ddot{x}}^{n+\frac{1}{2}}, u^{n+\frac{1}{2}}\right\rangle+2\left\langle\varphi\left(u^{n+\frac{1}{2}}\right), u^{n+\frac{1}{2}}\right\rangle \\
& -2\left\langle\kappa\left(u^{n+\frac{1}{2}}\right), u^{n+\frac{1}{2}}\right\rangle=0,
\end{aligned}
$$

where

$$
\varphi\left(u_{j}^{n+\frac{1}{2}}\right)=\frac{4}{9}\left\{\left(u_{j}^{n+\frac{1}{2}}\right)\left(u_{j}^{n+\frac{1}{2}}\right)_{\hat{x}}+\left[\left(u_{j}^{n+\frac{1}{2}}\right)^{2}\right]_{\hat{x}}\right\}
$$

and

$$
\kappa\left(u_{j}^{n+\frac{1}{2}}\right)=\frac{1}{9}\left\{\left(u_{j}^{n+\frac{1}{2}}\right)\left(u_{j}^{n+\frac{1}{2}}\right)_{\ddot{x}}+\left[\left(u_{j}^{n+\frac{1}{2}}\right)^{2}\right]_{\ddot{x}}\right\} .
$$

Since

$$
\begin{aligned}
\left\langle u_{\hat{x}}^{n+\frac{1}{2}}, u^{n+\frac{1}{2}}\right\rangle=0, & \left\langle u_{\ddot{x}}^{n+\frac{1}{2}}, u^{n+\frac{1}{2}}\right\rangle=0, \\
\left\langle\varphi\left(u^{n+\frac{1}{2}}\right), u^{n+\frac{1}{2}}\right\rangle= & \frac{4}{9} h \sum_{j=0}^{J}\left\{u_{j}^{n+\frac{1}{2}}\left(u_{j}^{n+\frac{1}{2}}\right)_{\hat{x}}+\left[\left(u_{j}^{n+\frac{1}{2}}\right)^{2}\right]_{\hat{x}}\right\} u_{j}^{n+\frac{1}{2}} \\
& =\frac{4}{9} h \sum_{j=0}^{J}\left(u_{j}^{n+\frac{1}{2}}\right)^{2}\left(u_{j}^{n+\frac{1}{2}}\right)_{\hat{x}}+\frac{4}{9} h \sum_{j=0}^{J}\left[\left(u_{j}^{n+\frac{1}{2}}\right)^{2}\right]_{\hat{x}} u_{j}^{n+\frac{1}{2}} \\
& =\frac{4}{9} h \sum_{j=0}^{J}\left(u_{j}^{n+\frac{1}{2}}\right)^{2}\left(u_{j}^{n+\frac{1}{2}}\right)_{\hat{x}}-\frac{4}{9} h \sum_{j=0}^{J}\left(u_{j}^{n+\frac{1}{2}}\right)^{2}\left(u_{j}^{n+\frac{1}{2}}\right)_{\hat{x}} \\
& =0
\end{aligned}
$$


and

$$
\begin{aligned}
\left\langle\kappa\left(u^{n+\frac{1}{2}}\right), u^{n+\frac{1}{2}}\right\rangle & =\frac{1}{9} h \sum_{j=0}^{J}\left\{\left(u_{j}^{n+\frac{1}{2}}\right)\left(u_{j}^{n+\frac{1}{2}}\right)_{\ddot{x}}+\left[\left(u_{j}^{n+\frac{1}{2}}\right)^{2}\right]_{\ddot{x}}\right\} u_{j}^{n+\frac{1}{2}} \\
& =\frac{1}{9} h \sum_{j=0}^{J}\left(u_{j}^{n+\frac{1}{2}}\right)^{2}\left(u_{j}^{n+\frac{1}{2}}\right)_{\ddot{x}}+\frac{1}{9} h \sum_{j=0}^{J}\left[\left(u_{j}^{n+\frac{1}{2}}\right)^{2}\right]_{\ddot{x}} u_{j}^{n+\frac{1}{2}} \\
& =\frac{1}{9} h \sum_{j=0}^{J}\left(u_{j}^{n+\frac{1}{2}}\right)^{2}\left(u_{j}^{n+\frac{1}{2}}\right)_{\ddot{x}}-\frac{1}{9} h \sum_{j=0}^{J}\left(u_{j}^{n+\frac{1}{2}}\right)^{2}\left(u_{j}^{n+\frac{1}{2}}\right)_{\ddot{x}} \\
& =0 .
\end{aligned}
$$

Substituting (2.8)-(2.10) into (2.7), we have

$$
\left(\left\|u^{n+1}\right\|^{2}-\left\|u^{n}\right\|^{2}\right)+\frac{4}{3}\left(\left\|u_{x}^{n+1}\right\|^{2}-\left\|u_{x}^{n}\right\|^{2}\right)-\frac{1}{3}\left(\left\|u_{\hat{x}}^{n+1}\right\|^{2}-\left\|u_{\hat{x}}^{n}\right\|^{2}\right)=0
$$

Similarly, by the definition of $E^{n},(2.5)$ is obtained from (2.11).

\section{Existence}

To prove the existence of solution for scheme (2.1)-(2.3), the following Browder fixed point theorem should be introduced. For the proof, see [19].

Lemma 3.1 Let $H$ be a finite dimensional inner product space. Suppose that $g: H \rightarrow H$ is continuous, and there exists an $\alpha>0$ such that $\langle g(x), x\rangle>0$ for all $x \in H$ with $\|x\|=\alpha$. Then there exists $x^{*} \in H$ such that $g\left(x^{*}\right)=0$ and $\left\|x^{*}\right\| \leq \alpha$.

Theorem 3.1 There exists $u^{n} \in Z_{h}^{0}$ satisfying difference scheme (2.1)-(2.3).

Proof Use the mathematical induction. Obviously, with condition (2.2), the solution exists for $n=0$. Suppose that for $n \leq N-1, u^{0}, u^{1}, \ldots, u^{n}$ satisfy (2.1)-(2.3), then we prove that there exists $u^{n+1}$ satisfying (2.1)-(2.3).

Define an operator $g$ on $Z_{h}^{0}$ as follows:

$$
g(v)=2 v-2 u^{n}-\frac{8}{3} v_{x \bar{x}}+\frac{8}{3} u_{x \bar{x}}^{n}+\frac{2}{3} v_{\hat{x} \hat{x}}-\frac{2}{3} u_{\hat{x} \hat{x}}^{n}+\frac{4}{3} \tau v_{\hat{x}}-\frac{1}{3} \tau v_{\ddot{x}}+\tau \varphi(v)-\tau \kappa(v) .
$$

Taking the inner product of (3.1) with $v$, we get

$$
\left\langle v_{\hat{x}}, v\right\rangle=0, \quad\left\langle v_{\ddot{x}}, v\right\rangle=0, \quad\langle\varphi(v), v\rangle=0, \quad\langle\kappa(v), v\rangle=0 .
$$

From Lemma 2.1 and Cauchy-Schwarz inequality, we get

$$
\begin{aligned}
\langle g(v), v\rangle= & 2\|v\|^{2}-2\left\langle u^{n}, v\right\rangle+\frac{8}{3}\left\|v_{x}\right\|^{2}-\frac{8}{3}\left\langle u_{x}^{n}, v_{x}\right\rangle-\frac{2}{3}\left\|v_{\hat{x}}\right\|^{2}+\frac{2}{3}\left\langle u_{\hat{x}}^{n}, v_{\hat{x}}\right\rangle \\
\geq & 2\|v\|^{2}-\left(\|u\|^{2}+\|v\|^{2}\right)+\frac{8}{3}\left\|v_{x}\right\|^{2}-\frac{4}{3}\left(\left\|u_{x}^{n}\right\|^{2}+\left\|v_{x}\right\|^{2}\right) \\
& -\frac{2}{3}\left\|v_{\hat{x}}\right\|^{2}-\frac{1}{3}\left(\left\|u_{\hat{x}}^{n}\right\|^{2}+\left\|v_{\hat{x}}\right\|^{2}\right)
\end{aligned}
$$




$$
\begin{aligned}
& \geq\|v\|^{2}-\left\|u^{n}\right\|^{2}+\frac{1}{3}\left\|v_{x}\right\|^{2}-\frac{4}{3}\left\|u_{x}^{n}\right\|^{2}-\frac{1}{3}\left\|u_{\hat{x}}^{n}\right\|^{2} \\
& \geq\|v\|^{2}-\left(\left\|u^{n}\right\|^{2}+\frac{4}{3}\left\|u_{x}^{n}\right\|^{2}+\frac{1}{3}\left\|u_{\hat{x}}^{n}\right\|^{2}\right) .
\end{aligned}
$$

Hence, for $\forall v \in Z_{h}^{0},\langle g(v), v\rangle \geq 0$ when $\|v\|^{2}=\left(\left\|u^{n}\right\|^{2}+\frac{4}{3}\left\|u_{x}^{n}\right\|^{2}+\frac{1}{3}\left\|u_{\hat{x}}^{n}\right\|^{2}\right)+1$. By Lemma 3.1, there exists $v^{*} \in Z_{h}^{0}$ which satisfies $g\left(v^{*}\right)=0$. Let $u^{n+1}=2 v^{*}-u^{n}$, and it can be proved easily that $u^{n+1}$ is the solution of scheme (2.1)-(2.3).

\section{Priori estimate, convergence and unconditional stability}

Let $v(x, t)$ be the solution of problems (1.1)-(1.3) and $v_{j}^{n}=v\left(x_{j}, t_{n}\right)$, then the truncation error of scheme (2.1)-(2.3) is obtained as follows:

$$
\begin{aligned}
r_{j}^{n} & =\left(v_{j}^{n}\right)_{t}-\frac{4}{3}\left(v_{j}^{n}\right)_{x \bar{x} t}+\frac{1}{3}\left(v_{j}^{n}\right)_{\hat{x} \hat{x} t}+\frac{4}{3}\left(v_{j}^{n+\frac{1}{2}}\right)_{\hat{x}}-\frac{1}{3}\left(v_{j}^{n+\frac{1}{2}}\right)_{\ddot{x}}++\varphi\left(v_{j}^{n+\frac{1}{2}}\right)-\kappa\left(v_{j}^{n+\frac{1}{2}}\right), \\
j & =1,2, \ldots, J-1 ; n=1,2, \ldots, N-1, \\
v_{j}^{0} & =u_{0}\left(x_{j}\right), \quad 0 \leq j \leq J, \\
v^{n} & \in Z_{h}^{0}, \quad n=0,1,2, \ldots, N .
\end{aligned}
$$

According to Taylor expansion, we obtain the following result.

Theorem 4.1 $\left|r_{j}^{n}\right|=O\left(\tau^{2}+h^{4}\right)$ holds as $\tau, h \rightarrow 0$.

Proof Since $v(x, t)$ is the solution of problems (1.1)-(1.3), we have

$$
v_{t}+v_{x}+v v_{x}-v_{x x t}=0, \quad x \in\left(x_{L}, x_{R}\right), t \in(0, T] .
$$

Firstly, considering the term $v_{t}$, by Taylor expansion at the point $\left(x_{j}, t_{n+\frac{1}{2}}\right)$, we get

$$
\begin{aligned}
& v_{j}^{n+1}=v_{j}^{n+\frac{1}{2}}+\left.\left(\frac{\tau}{2}\right) v_{t}\right|_{j} ^{n+\frac{1}{2}}+\left.\frac{1}{2 !}\left(\frac{\tau}{2}\right)^{2} v_{t t}\right|_{j} ^{n+\frac{1}{2}}+O\left(\tau^{3}\right), \\
& v_{j}^{n}=v_{j}^{n+\frac{1}{2}}+\left.\left(-\frac{\tau}{2}\right) v_{t}\right|_{j} ^{n+\frac{1}{2}}+\left.\frac{1}{2 !}\left(-\frac{\tau}{2}\right)^{2} v_{t t}\right|_{j} ^{n+\frac{1}{2}}+O\left(\tau^{3}\right) .
\end{aligned}
$$

It follows from (4.5) and (4.6) that

$$
\left.v_{t}\right|_{j} ^{n+\frac{1}{2}}=\frac{v_{j}^{n+1}-v_{j}^{n}}{\tau}+O\left(\tau^{2}\right)=\left(v_{j}^{n}\right)_{t}+O\left(\tau^{2}\right) .
$$

Similarly, by Taylor expansion, we can obtain the following results, respectively:

$$
\begin{aligned}
\left.\left(v_{x}\right)\right|_{j} ^{n+\frac{1}{2}} & =\frac{v_{j+1}^{n+\frac{1}{2}}-v_{j-1}^{n+\frac{1}{2}}}{2 h}-\left.\frac{1}{6} h^{2}\left(v_{x x x}\right)\right|_{j} ^{n+\frac{1}{2}}+O\left(h^{4}\right) \\
& =\left(v_{j}^{n+\frac{1}{2}}\right)_{\hat{x}}-\left.\frac{1}{6} h^{2}\left(v_{x x x}\right)\right|_{j} ^{n+\frac{1}{2}}+O\left(h^{4}\right),
\end{aligned}
$$




$$
\begin{aligned}
\left.\left(v_{x}\right)\right|_{j} ^{n+\frac{1}{2}} & =\frac{v_{j+2}^{n+\frac{1}{2}}-v_{j-2}^{n+\frac{1}{2}}}{4 h}-\left.\frac{2}{3} h^{2}\left(v_{x x x}\right)\right|_{j} ^{n+\frac{1}{2}}+O\left(h^{4}\right) \\
& =\left(v_{j}^{n+\frac{1}{2}}\right)_{\ddot{x}}-\left.\frac{2}{3} h^{2}\left(v_{x x x}\right)\right|_{j} ^{n+\frac{1}{2}}+O\left(h^{4}\right)
\end{aligned}
$$

and

$$
\begin{aligned}
& \left.\left(v_{x x}\right)\right|_{j} ^{n+\frac{1}{2}}=\left(v_{j}^{n+\frac{1}{2}}\right)_{x \bar{x}}-\left.\frac{1}{12} h^{2}\left(v_{x x x x}\right)\right|_{j} ^{n+\frac{1}{2}}+O\left(h^{4}\right), \\
& \left.\left(v_{x x}\right)\right|_{j} ^{n+\frac{1}{2}}=\left(v_{j}^{n+\frac{1}{2}}\right)_{\hat{x} \hat{x}}-\left.\frac{1}{3} h^{2}\left(v_{x x x x}\right)\right|_{j} ^{n+\frac{1}{2}}+O\left(h^{4}\right) .
\end{aligned}
$$

Thus, by (4.8) and (4.9), we have

$$
\frac{4}{3}\left(v_{j}^{n+\frac{1}{2}}\right)_{\hat{x}}-\frac{1}{3}\left(v_{j}^{n+\frac{1}{2}}\right)_{\ddot{x}}=\left.\left(v_{x}\right)\right|_{j} ^{n+\frac{1}{2}}+O\left(h^{4}\right) .
$$

By (4.10) and (4.11), we have

$$
\frac{4}{3}\left(v_{j}^{n+\frac{1}{2}}\right)_{x \bar{x}}-\frac{1}{3}\left(v_{j}^{n+\frac{1}{2}}\right)_{\hat{x} \hat{x}}=\left.\left(v_{x x}\right)\right|_{j} ^{n+\frac{1}{2}}+O\left(h^{4}\right) .
$$

Moreover,

$$
\begin{aligned}
\varphi & \left(v_{j}^{n+\frac{1}{2}}\right)-\kappa\left(v_{j}^{n+\frac{1}{2}}\right) \\
& =\frac{4}{9}\left\{\left(v_{j}^{n+\frac{1}{2}}\right)\left(v_{j}^{n+\frac{1}{2}}\right)_{\hat{x}}+\left[\left(v_{j}^{n+\frac{1}{2}}\right)^{2}\right]_{\hat{x}}\right\}-\frac{1}{9}\left\{v_{j}^{n+\frac{1}{2}}\left(v_{j}^{n+\frac{1}{2}}\right)_{\ddot{x}}+\left[\left(v_{j}^{n+\frac{1}{2}}\right)^{2}\right]_{\ddot{x}}\right\} \\
& =\frac{1}{3} v_{j}^{n+\frac{1}{2}}\left\{\frac{4}{3}\left(u_{j}^{n+\frac{1}{2}}\right)_{\hat{x}}-\frac{1}{3}\left(u_{j}^{n+\frac{1}{2}}\right)_{\ddot{x}}\right\}+\frac{1}{3}\left\{\frac{4}{3}\left[\left(u_{j}^{n+\frac{1}{2}}\right)^{2}\right]_{\hat{x}}-\frac{1}{3}\left[\left(u_{j}^{n+\frac{1}{2}}\right)^{2}\right]_{\ddot{x}}\right\} \\
& =\left.\left(u u_{x}\right)\right|_{j} ^{n+\frac{1}{2}}+O\left(h^{4}\right) .
\end{aligned}
$$

Apparently, it follows from (4.7), (4.12), (4.13) and (4.14) that (4.4) holds.

Lemma 4.1 Suppose that $u_{0} \in H_{0}^{1}\left[x_{L}, x_{R}\right]$, then the solution of the initial-boundary value problems (1.1)-(1.3) satisfies

$$
\|u\|_{L_{2}} \leq C, \quad\left\|u_{x}\right\|_{L_{2}} \leq C, \quad\|u\|_{L_{\infty}} \leq C
$$

Proof It follows from (1.6) that

$$
E(t)=\|u\|_{L_{2}}^{2}+\left\|u_{x}\right\|_{L_{2}}^{2}=E(0)=C,
$$

which yields

$$
\|u\|_{L_{2}} \leq C, \quad\left\|u_{x}\right\|_{L_{2}} \leq C .
$$

By Sobolev inequality, $\|u\|_{L_{\infty}} \leq C$ holds. 
Lemma 4.2 Suppose that $u_{0} \in H_{0}^{1}\left[x_{L}, x_{R}\right]$, then the solution of scheme (2.1)-(2.3) satisfies

$$
\left\|u^{n}\right\| \leq C, \quad\left\|u_{x}^{n}\right\| \leq C, \quad\left\|u^{n}\right\|_{\infty} \leq C
$$

for $n=1,2, \ldots, N$.

Proof It follows from Theorem 2.1 and Lemma 2.1 that

$$
\left\|u^{n}\right\|^{2}+\left\|u_{x}^{n}\right\|^{2} \leq E^{n}=\left\|u^{n}\right\|^{2}+\frac{4}{3}\left\|u_{x}^{n}\right\|^{2}-\frac{1}{3}\left\|u_{\hat{x}}^{n}\right\|^{2}=C,
$$

that is,

$$
\left\|u^{n}\right\| \leq C, \quad\left\|u_{x}^{n}\right\| \leq C
$$

By discrete Sobolev inequality [18], we have $\left\|u^{n}\right\|_{\infty} \leq C$.

Theorem 4.2 Suppose that $u_{0} \in H_{0}^{1}\left[x_{L}, x_{R}\right]$, then the solution $u^{n}$ of difference scheme (2.1)(2.3) converges to the solution of problems (1.1)-(1.3) with order $O\left(\tau^{2}+h^{4}\right)$ by the $\|\cdot\|_{\infty}$ norm.

Proof Letting

$$
e_{j}^{n}=v_{j}^{n}-u_{j}^{n},
$$

and subtracting (2.1)-(2.3) from (4.1)-(4.3), respectively, we have

$$
\begin{aligned}
r_{j}^{n}= & \left(e_{j}^{n}\right)_{t}-\frac{4}{3}\left(e_{j}^{n}\right)_{x \bar{x} t}+\frac{1}{3}\left(e_{j}^{n}\right)_{\hat{x} \hat{x} t}+\frac{4}{3}\left(e_{j}^{n+\frac{1}{2}}\right)_{\hat{x}} \\
& -\frac{1}{3}\left(e_{j}^{n+\frac{1}{2}}\right)_{\ddot{x}}+\varphi\left(v_{j}^{n+\frac{1}{2}}\right)-\varphi\left(u_{j}^{n+\frac{1}{2}}\right) \\
& -\kappa\left(v_{j}^{n+\frac{1}{2}}\right)+\kappa\left(u_{j}^{n+\frac{1}{2}}\right), \quad j=1,2, \ldots, J-1 ; n=1,2, \ldots, N-1, \\
e_{j}^{0}= & 0, \quad 0 \leq j \leq J, \\
e^{n} \in & Z_{h}^{0}, \quad n=0,1,2, \ldots, N .
\end{aligned}
$$

Computing the inner product of (4.15) with $2 e^{n+\frac{1}{2}}$, and using boundary condition (4.17), we get

$$
\begin{aligned}
\left\langle r^{n}, 2 e^{n+\frac{1}{2}}\right\rangle= & \left\|e^{n}\right\|_{t}^{2}+\frac{4}{3}\left\|e_{x}^{n}\right\|_{t}^{2}-\frac{1}{3}\left\|e_{\hat{x}}^{n}\right\|_{t}^{2}+\frac{8}{3}\left\langle e_{\hat{x}}^{n+\frac{1}{2}}, e^{n+\frac{1}{2}}\right\rangle-\frac{2}{3}\left\langle e_{\ddot{x}}^{n+\frac{1}{2}}, e^{n+\frac{1}{2}}\right\rangle \\
& +2\left\langle\varphi\left(v^{n+\frac{1}{2}}\right)-\varphi\left(u^{n+\frac{1}{2}}\right), e^{n+\frac{1}{2}}\right\rangle-2\left\langle\kappa\left(v^{n+\frac{1}{2}}\right)-\kappa\left(u^{n+\frac{1}{2}}\right), e^{n+\frac{1}{2}}\right\rangle .
\end{aligned}
$$

Similarly to (2.8), we have

$$
\left\langle e_{\hat{x}}^{n+\frac{1}{2}}, e^{n+\frac{1}{2}}\right\rangle=0, \quad\left\langle e_{\ddot{x}}^{n+\frac{1}{2}}, e^{n+\frac{1}{2}}\right\rangle=0
$$


Zheng and Mu Advances in Difference Equations 2013, 2013:287

Page 9 of 12

According to Lemma 4.1, Lemma 4.2, Theorem 2.1 and Cauchy-Schwartz inequality, we get

$$
\begin{aligned}
\langle\varphi & \left.\left(v^{n+\frac{1}{2}}\right)-\varphi\left(u^{n+\frac{1}{2}}\right), e^{n+\frac{1}{2}}\right\rangle \\
& =\frac{4}{9} h \sum_{j=0}^{J}\left[v_{j}^{n+\frac{1}{2}}\left(v_{j}^{n+\frac{1}{2}}\right)_{\hat{x}}-u_{j}^{n+\frac{1}{2}}\left(u_{j}^{n+\frac{1}{2}}\right)_{\hat{x}}\right] e_{j}^{n+\frac{1}{2}}+\frac{4}{9} h \sum_{j=0}^{J}\left[\left(v_{j}^{n+\frac{1}{2}}\right)^{2}-\left(u_{j}^{n+\frac{1}{2}}\right)^{2}\right]_{\hat{x}} e_{j}^{n+\frac{1}{2}} \\
& =\frac{4}{9} h \sum_{j=0}^{J}\left[v_{j}^{n+\frac{1}{2}}\left(e_{j}^{n+\frac{1}{2}}\right)_{\hat{x}}+e_{j}^{n+\frac{1}{2}}\left(u_{j}^{n+\frac{1}{2}}\right)_{\hat{x}}\right] e_{j}^{n+\frac{1}{2}}-\frac{4}{9} h \sum_{j=0}^{J}\left[e_{j}^{n+\frac{1}{2}}\left(u_{j}^{n+\frac{1}{2}}+v_{j}^{n+\frac{1}{2}}\right)\right]\left(e_{j}^{n+\frac{1}{2}}\right)_{\hat{x}} \\
& \leq C\left(\left\|e^{n+1}\right\|^{2}+\left\|e^{n}\right\|^{2}+\left\|e_{\hat{x}}^{n+1}\right\|^{2}+\left\|e_{\hat{x}}^{n}\right\|^{2}\right) \\
& \leq C\left(\left\|e^{n+1}\right\|^{2}+\left\|e^{n}\right\|^{2}+\left\|e_{x}^{n+1}\right\|^{2}+\left\|e_{x}^{n}\right\|^{2}\right), \\
\langle\kappa & \left.\left(v^{n+\frac{1}{2}}\right)-\kappa\left(u^{n+\frac{1}{2}}\right), e^{n+\frac{1}{2}}\right\rangle \\
& =\frac{1}{9} h \sum_{j=0}^{J}\left[v_{j}^{n+\frac{1}{2}}\left(v_{j}^{n+\frac{1}{2}}\right)_{\ddot{x}}-u_{j}^{n+\frac{1}{2}}\left(u_{j}^{n+\frac{1}{2}}\right)_{\ddot{x}}\right] e_{j}^{n+\frac{1}{2}}+\frac{1}{9} h \sum_{j=0}^{J}\left[\left(v_{j}^{n+\frac{1}{2}}\right)^{2}-\left(u_{j}^{n+\frac{1}{2}}\right)^{2}\right]_{\ddot{x}} e_{j}^{n+\frac{1}{2}} \\
& =\frac{1}{9} h \sum_{j=0}^{J}\left[v_{j}^{n+\frac{1}{2}}\left(e_{j}^{n+\frac{1}{2}}\right)_{\ddot{x}}+e_{j}^{n+\frac{1}{2}}\left(u_{j}^{n+\frac{1}{2}}\right)_{\ddot{x}}\right] e_{j}^{n+\frac{1}{2}}-\frac{1}{9} h \sum_{j=0}^{J}\left[e_{j}^{n+\frac{1}{2}}\left(u_{j}^{n+\frac{1}{2}}+v_{j}^{n+\frac{1}{2}}\right)\right]\left(e_{j}^{n+\frac{1}{2}}\right)_{\ddot{x}} \\
& \leq C\left(\left\|e^{n+1}\right\|^{2}+\left\|e^{n}\right\|^{2}+\left\|e_{\ddot{x}}^{n+1}\right\|^{2}+\left\|e_{\ddot{x}}^{n}\right\|^{2}\right) \\
& \leq C\left(\left\|e^{n+1}\right\|^{2}+\left\|e^{n}\right\|^{2}+\left\|e_{x}^{n+1}\right\|^{2}+\left\|e_{x}^{n}\right\|^{2}\right)
\end{aligned}
$$

and

$$
\left\langle r^{n}, 2 e^{n+\frac{1}{2}}\right\rangle=\left\langle r^{n}, e^{n+1}+e^{n}\right\rangle \leq\left\|r^{n}\right\|^{2}+\left\|e^{n+1}\right\|^{2}+\left\|e^{n}\right\|^{2}
$$

Substituting (4.19)-(4.22) into (4.18), we get

$$
\left\|e^{n}\right\|_{t}^{2}+\frac{4}{3}\left\|e_{x}^{n}\right\|_{t}^{2}-\frac{1}{3}\left\|e_{\hat{x}}^{n}\right\|_{t}^{2} \leq\left\|r^{n}\right\|^{2}+C\left(\left\|e^{n+1}\right\|^{2}+\left\|e^{n}\right\|^{2}+\left\|e_{x}^{n+1}\right\|^{2}+\left\|e_{x}^{n}\right\|^{2}\right) .
$$

Letting $B^{n}=\left\|e^{n}\right\|^{2}+\frac{4}{3}\left\|e_{x}^{n}\right\|^{2}-\frac{1}{3}\left\|e_{\hat{x}}^{n}\right\|^{2}$ and summing up (4.23) from 0 to $n-1$, we have

$$
B^{n} \leq B^{0}+C \tau \sum_{l=0}^{n-1}\left\|r^{l}\right\|^{2}+C \tau \sum_{l=0}^{n}\left(\left\|e^{l}\right\|^{2}+\left\|e_{x}^{l}\right\|^{2}\right) .
$$

Noticing

$$
\tau \sum_{l=0}^{n-1}\left\|r^{l}\right\|^{2} \leq n \tau \max _{0 \leq l \leq n-1}\left\|r^{l}\right\|^{2} \leq T \cdot O\left(\tau^{2}+h^{4}\right)^{2}
$$

and $B^{0}=O\left(\tau^{2}+h^{4}\right)^{2}$, from (4.24), we get

$$
\left\|e^{n}\right\|^{2}+\left\|e_{x}^{n}\right\|^{2} \leq B^{n} \leq O\left(\tau^{2}+h^{4}\right)^{2}+C \tau \sum_{l=0}^{n}\left(\left\|e^{l}\right\|^{2}+\left\|e_{x}^{l}\right\|^{2}\right) .
$$


By discrete Gronwall inequality [18], we have

$$
\left\|e^{n}\right\| \leq O\left(\tau^{2}+h^{4}\right), \quad\left\|e_{x}^{n}\right\| \leq O\left(\tau^{2}+h^{4}\right) .
$$

Finally, by discrete Sobolev inequality [18], we get

$$
\left\|e^{n}\right\|_{\infty} \leq O\left(\tau^{2}+h^{4}\right)
$$

This completes the proof of Theorem 4.2.

Similarly, we can prove the stability and uniqueness of the difference solution.

Theorem 4.3 Under the conditions of Theorem 4.2, the solution of scheme (2.1)-(2.3) is stable by the $\|\cdot\|_{\infty}$ norm.

Theorem 4.4 The solution $u^{n}$ of scheme (2.1)-(2.3) is unique.

\section{Numerical experiments}

In this section, we compute a numerical example to demonstrate the effectiveness of our difference scheme. The single solitary-wave solution of RLW equation (1.1) is given by

$$
u(x, t)=A \operatorname{sech}^{2}(k x-\omega t+\delta)
$$

where

$$
A=\frac{3 a^{2}}{1-a^{2}}, \quad k=\frac{a}{2}, \quad \omega=\frac{a}{2\left(1-a^{2}\right)},
$$

and $a, \delta$ are constants.

Scheme (2.1)-(2.3) is a nonlinear system of equations which can be solved by the Newton iteration. Take $a=\frac{1}{2}, \delta=0$, and the initial function of problems (1.1)-(1.3) is rewritten as

$$
u(x, 0)=\operatorname{sech}^{2}\left(\frac{1}{4} x\right)
$$

In the numerical experiments, we take $x_{L}=-50, x_{R}=50$ and $T=10$. The errors in the

\begin{tabular}{|c|c|c|c|c|c|c|}
\hline & \multicolumn{2}{|c|}{$\tau=0.2, h=0.1$} & \multicolumn{2}{|c|}{$\tau=0.05, h=0.05$} & \multicolumn{2}{|c|}{$\tau=0.0125, h=0.025$} \\
\hline & $\left\|e^{n}\right\|$ & $\left\|e^{n}\right\|_{\infty}$ & $\left\|e^{n}\right\|$ & $\left\|e^{n}\right\|_{\infty}$ & $\left\|e^{n}\right\|$ & $\left\|e^{n}\right\|_{\infty}$ \\
\hline$t=2$ & $1.815667 \mathrm{e}-3$ & $9.106257 \mathrm{e}-$ & $1.139049 \mathrm{e}-4$ & $5.714576 e-6$ & 7.120731 & $3.572519 \mathrm{e}-6$ \\
\hline$t=4$ & $3.558495 \mathrm{e}-3$ & $1.786774 \mathrm{e}-3$ & $2.232765 \mathrm{e}-4$ & $1.121945 \mathrm{e}-4$ & $1.395820 \mathrm{e}-5$ & $7.013983 e-6$ \\
\hline$t=6$ & $5.186555 \mathrm{e}-3$ & $2.536722 \mathrm{e}-3$ & $3.254965 e-4$ & $1.592516 \mathrm{e}-4$ & $2.034878 e-5$ & $9.955996 \mathrm{e}-6$ \\
\hline$t=8$ & $6.691763 e-3$ & $3.175770 \mathrm{e}-3$ & $4.200524 \mathrm{e}-4$ & $1.994228 \mathrm{e}-4$ & $2.626042 e-5$ & $1.246776 \mathrm{e}-5$ \\
\hline$t=10$ & $8.084741 \mathrm{e}-3$ & $3.734968 \mathrm{e}-3$ & $5.076006 \mathrm{e}-4$ & $2.346426 \mathrm{e}-4$ & $3.173410 \mathrm{e}-5$ & $1.467109 e-5$ \\
\hline
\end{tabular}
sense of $L_{\infty}$-norm and $L_{2}$-norm of the numerical solutions under different mesh steps $h$ and $\tau$ are listed in Table 1 . Table 2 shows that the computational and the theoretical orders of the scheme are very close to each other. Furthermore, since we have shown in

Table 1 The errors estimates of numerical solution with various $h$ and $\tau$ 
Table 2 The numerical verification of theoretical accuracy $O\left(\tau^{2}+h^{4}\right)$

\begin{tabular}{|c|c|c|c|c|c|c|}
\hline & \multicolumn{3}{|c|}{$\left\|e^{n}(h, \tau)\right\| /\left\|e^{4 n}\left(\frac{h}{2}, \frac{\tau}{4}\right)\right\|$} & \multicolumn{3}{|c|}{$\left\|e^{n}(h, \tau)\right\|_{\infty} /\left\|e^{4 n}\left(\frac{h}{2}, \frac{\tau}{4}\right)\right\|_{\infty}$} \\
\hline & $\begin{aligned} \tau & =0.2, \\
h & =0.1\end{aligned}$ & $\begin{aligned} \tau & =0.05 \\
h & =0.05\end{aligned}$ & $\begin{array}{l}\tau=0.0125 \\
h=0.025\end{array}$ & $\begin{array}{l}\tau=0.2 \\
h=0.1\end{array}$ & $\begin{aligned} \tau & =0.05 \\
h & =0.05\end{aligned}$ & $\begin{aligned} \tau & =0.0125, \\
h & =0.025\end{aligned}$ \\
\hline$t=2$ & - & 15.940202 & 15.996241 & - & 15.935139 & 15.995926 \\
\hline$t=4$ & - & 15.937612 & 15.996078 & - & 15.925675 & 15.995839 \\
\hline$t=6$ & - & 15.934289 & 15.995869 & - & 15.929016 & 15.995554 \\
\hline$t=8$ & - & 15.930779 & 15.995648 & - & 15.924811 & 15.995067 \\
\hline$t=10$ & - & 15.927366 & 15.995430 & - & 15.917690 & 15.993525 \\
\hline
\end{tabular}

Table 3 Discrete mass and discrete energy with various $h$ and $\tau$

\begin{tabular}{|c|c|c|c|c|}
\hline & \multicolumn{2}{|l|}{$\tau=0.2, h=0.1$} & \multicolumn{2}{|c|}{$\tau=0.05, h=0.05$} \\
\hline & $\overline{Q^{n}}$ & $E^{n}$ & $\overline{Q^{n}}$ & $E^{n}$ \\
\hline$t=2$ & 7.99999999942 & 5.59999998149 & 7.99999999938 & 5.59999999884 \\
\hline$t=4$ & 7.99999999815 & 5.59999998149 & 7.99999999797 & 5.59999999884 \\
\hline$t=6$ & 7.99999999320 & 5.59999998149 & 7.99999999255 & 5.59999999884 \\
\hline$t=8$ & 7.99999997441 & 5.59999998149 & 7.99999997199 & 5.59999999884 \\
\hline$t=10$ & 7.99999990304 & 5.59999998149 & 7.99999999404 & 5.59999999884 \\
\hline
\end{tabular}

Theorem 2.1 that the numerical solution $u^{n}$ satisfies invariants (2.4) and (2.5), respectively, Table 3 is also presented to show the conservative laws of discrete mass $Q^{n}$ and discrete energy $E^{n}$.

From these computational results, the stability and convergence of the scheme are verified, and it shows that our proposed algorithm is effective.

Competing interests

The authors declare that they have no competing interests.

Authors' contributions

The paper is a joint work of all authors who contributed equally to the final version of the paper. All authors read and approved the final manuscript.

\section{Author details}

${ }^{1}$ School of Science, Southwest University of Science and Technology, Mianyang, Sichuan 621010, P.R. China. ${ }^{2}$ School of Mathematics and Computer Engineering, Xihua University, Chengdu, Sichuan 610039, P.R. China.

\section{Acknowledgements}

The authors would like to thank the editor and the anonymous reviewers for their constructive comments and suggestions to improve the quality of the paper. This work is supported by the Scientific Research Fund of Sichuan Provincial Education Department (No. 11ZB009) and the Doctoral Program Research Fund of Southwest University of Science and Technology (No. 11zx7129).

Received: 21 July 2013 Accepted: 11 September 2013 Published: 07 Nov 2013

\section{References}

1. Peregrine, DH: Long waves on beach. J. Fluid Mech. 27, 815-827 (1967)

2. Bona, JL, Bryant, PJ: A mathematical model for long waves generated by wave makers in nonlinear dispersive systems. Proc. Camb. Philos. Soc. 73, 391-405 (1973)

3. Wazwaz, AM: Analytic study on nonlinear variants of the RLW and the PHI-four equations. Commun. Nonlinear Sci. Numer. Simul. 12, 314-327 (2007)

4. Soliman, AA: Numerical simulation of the generalized regularized long wave equation by He's variational iteration method. Math. Comput. Simul. 70, 119-124 (2005)

5. Yusufoglu, E, Bekir, A: Application of the variational iteration method to the regularized long wave equation. Comput. Math. Appl. 54, 1154-1161 (2007)

6. Chang, Q, Wang, G, Guo, B: Conservative scheme for a model of nonlinear dispersive waves and its solitary waves induced by boundary motion. J. Comput. Phys. 93, 360-375 (1991)

7. Ramos, J: Explicit finite difference methods for the EW and RLW equations. Appl. Math. Comput. 179, 622-638 (2006)

8. Zhang, L, Chang, Q: A new finite difference method for regularized long wave equation. J. Numer. Methods Comput. Appl. 21, 247-254 (2000)

9. Christou, MA, Christov, Cl: Interacting localized waves for the regularized long wave equation via a Galerkin spectral method. Math. Comput. Simul. 69, 257-268 (2005) 
10. Alexender, ME, Morris, JL: Galerkin method applied to some model equations for nonlinear dispersive waves. J. Comput. Phys. 30, 428-451 (1979)

11. Esen, A, Kutluay, S: Application of a lumped Galerkin method to the regularized long wave equation. Appl. Math. Comput. 174, 833-845 (2006)

12. Gu, H, Chen, N: Least-squares mixed finite element methods for the RLW equations. Numer. Methods Partial Differ Equ. 24, 749-758 (2008)

13. Mei, L, Chen, Y: Numerical solutions of RLW equation using Galerkin method with extrapolation techniques. Comput. Phys. Commun. 183, 1609-1616 (2012)

14. Soliman, AA, Hussien, MH: Collocation solution for RLW equation with septic spline. Appl. Math. Comput. 161 623-636 (2005)

15. El-Danaf, TS, Ramadan, MA, Abd Alaal, FEl: The use of adomian decomposition method for solving the regularized long-wave equation. Chaos Solitons Fractals 26, 747-757 (2005)

16. Li, S, Vu-Quoc, L: Finite difference calculus invariant structure of a class of algorithms for the nonlinear Klein-Gordon equation. SIAM J. Numer. Anal. 32, 1839-1875 (1995)

17. Zhang, F, Victor, MP, Luis, V: Numerical simulation of nonlinear Schrödinger systems: a new conservative scheme. Appl. Math. Comput. 71, 165-177 (1995)

18. Zhou, Y: Application of Discrete Functional Analysis to the Finite Difference Method. Inter. Acad. Publishers, Beijing (1990)

19. Browder, FE: Existence and uniqueness theorems for solutions of nonlinear boundary value problems. Proc. Symp. Appl. Math. 17, 24-49 (1965)

10.1186/1687-1847-2013-287

Cite this article as: Zheng and Hu: High-order conservative Crank-Nicolson scheme for regularized long wave equation. Advances in Difference Equations 2013, 2013:287

\section{Submit your manuscript to a SpringerOpen ${ }^{\circ}$ journal and benefit from:}

- Convenient online submission

- Rigorous peer review

- Immediate publication on acceptance

- Open access: articles freely available online

- High visibility within the field

- Retaining the copyright to your article 